\title{
Permeability, Permittivity and EM-wave Absorption Properties of Polymer Composites Filled with MnZn Ferrite and Carbon Black
}

\author{
R. Dosoudil ${ }^{a, *}$, K. LisÝ ${ }^{a}$ AND J. KRUŽElÁK ${ }^{b}$ \\ ${ }^{a}$ Institute of Electrical Engineering, Slovak University of Technology in Bratislava, \\ Ilkovičova 3, 81219 Bratislava, Slovakia \\ ${ }^{b}$ Institute of Polymer and Synthetic Materials, Slovak University of Technology in Bratislava, \\ Radlinského 9, 81237 Bratislava, Slovakia
}

\begin{abstract}
Composite materials based on manganese-zinc ferrite $(\mathrm{MnZn})$ and carbon black $(\mathrm{CB})$ as fillers, and natural butadiene rubber (NBR) as a polymeric non-magnetic matrix have been prepared by means of a hot-pressing route. Frequency dispersion of complex (relative) permeability $\mu$ and permittivity $\varepsilon$ was measured on composite samples using combined impedance/transmission line method and is ascribed to magnetic and dielectric loss in association with domain wall resonance, spin precession resonance, and interfacial polarization. Electromagnetic wave absorption characteristics of composites (such as return loss RL, matching thickness $d_{m}$, matching frequency $f_{m}$, bandwidth $\Delta f$ for $\mathrm{RL} \leq-20 \mathrm{~dB}$, and the minimum of return loss $\mathrm{RL}_{\min }$ ) were evaluated through numerical simulations depending on the relation between material parameters $(\mu, \varepsilon)$ and the content of hybrid $\mathrm{MnZn} / \mathrm{CB}$ filler. The synthesized composites appear to be excellent electromagnetic shields within the frequency interval $300 \mathrm{MHz}-2 \mathrm{GHz}$.
\end{abstract}

DOI: 10.12693/APhysPolA.137.827

PACS/topics: composite, ferrite, carbon black, permeability, permittivity, return loss

\section{Introduction}

Because of the fast expansion of modern electronic wireless communication and information technology systems, and due to an increase of electronic devices in printed circuit boards emitting unwanted electromagnetic noise, the designers and engineers of these systems and circuits are faced a problem of electromagnetic interference (EMI). To solve this problem, magneto-dielectric composite materials consisting of magneto-dielectric fillers (such as soft ferrites) and non-magnetic matrices (such as polymers) have been used. These materials can be good electromagnetic shields if they prevent both incoming and outgoing EMI noise. One can assure that with appropriate frequency dispersion of both the complex permeability and the complex permittivity at a desired frequency range. Permeability dispersion of composite is determined by magnetic filler only, while the permittivity is given by all composite constituents (filler and matrix). Electrical conductivity of constituents also may have a direct impact on the increase in permittivity of composite. The electrically conductive polymers can be used as matrices to enhance permittivity of composites, but these polymers are expensive and therefore less suitable. Probably the best choice is to

*corresponding author; e-mail: rastislav.dosoudil@stuba.sk embed a small amount of conductive filler such as carbon black (CB) into a non-conductive polymer matrix such as rubber. CB nanoparticles have the graphitic crystallite structure resulting in a good electrical conductivity $\left(\sigma_{D C} \approx 5-200 \mathrm{~S} / \mathrm{m}\right)$, and can also serve as a rubber reinforcement. In addition, the dielectric character of CB may contribute to higher electromagnetic wave (EM-wave) absorbing ability [1, 2]. Herein, MnZn ferrite and carbon black as powder fillers have been added into the natural butadiene rubber (NBR) polymer matrix to fabricate $\mathrm{MnZn} / \mathrm{CB} / \mathrm{NBR}$ composite materials. The magnetic, dielectric and high-frequency EM-wave absorbing performances of composite materials have been investigated as well.

\section{Experimental}

In this study, MnZn/CB/NBR composite materials based on manganese-zinc (MnZn) ferrite with composition $\approx \mathrm{Mn}_{0.68} \mathrm{Zn}_{0.24} \mathrm{Fe}_{2.08} \mathrm{O}_{4}$ and carbon black $(\mathrm{CB})$ as fillers, and natural butadiene rubber (NBR) as a polymeric non-magnetic matrix have been synthesized. The compound of rubber batch with the constant content of carbon black (20 phr) and variable content of MnZn ferrite (0-500 phr in steps of $100 \mathrm{phr}$ ) was obtained by hot-mixing at $90^{\circ} \mathrm{C}$. Then, the cross-linking of the compound was carried out for the optimum vulcanization time, at $160{ }^{\circ} \mathrm{C}$ and a pressure value of $15 \mathrm{MPa}$ using a hydraulic press. The composite samples were cut from final rubber sheets (with size $150 \mathrm{~mm} \times 150 \mathrm{~mm}$ ) 
into the rings (with an outer diameter of $7 \mathrm{~mm}$, an inner diameter of $3.05 \mathrm{~mm}$ and a thickness of $2 \mathrm{~mm}$ ), and into discs (with an outer diameter of $20 \mathrm{~mm}$ and a thickness of $2 \mathrm{~mm}$ ). The composite samples throughout the text and in figures are designated according to the content of MnZn ferrite, i.e., $500 \mathrm{phr}$, $400 \mathrm{phr}$, $300 \mathrm{phr}, 200 \mathrm{phr}, 100 \mathrm{phr}$, and 0 phr. The surface morphology and microstructure of composite materials were observed using scanning electron microscope JEOL JSM-7500F. The frequency dependences of complex permeability and permittivity of prepared composite materials have been measured in the range $1-3 \mathrm{GHz}$ by means of a combined impedance/transmission line method using a vector network analyser (Keysight E5063A). The DC electrical conductivity $\sigma_{\mathrm{DC}}$ of fillers $(\mathrm{MnZn}$ ferrite and carbon black) was determined using standard twoprobe method. Based on frequency responses of material parameters, the monolayer EM-wave absorption characteristics (such as return loss RL, matching thickness $d_{m}$, matching frequency $f_{m}$, bandwidth $\Delta f$ for $\mathrm{RL} \leq-20 \mathrm{~dB}$, and the minimum of return loss $\mathrm{RL}_{\min }$ ) were computed from return loss RL. It is defined as follows:

$$
\mathrm{RL}=20 \log \left|\frac{Z_{\text {in }}-1}{Z_{\text {in }}+1}\right|,
$$

where

$$
Z_{\text {in }}=\sqrt{\frac{\mu}{\varepsilon}} \tanh \left(\mathrm{i} \frac{\omega d}{c}(\mu \varepsilon)\right)
$$

is the normalized value of input complex impedance of the absorber, $\omega$ is the angular frequency, $d$ is the thickness of the monolayer absorber (backed by a metal sheet), and $c$ is the velocity of light in free space. The composite absorbs the maximum of the energy if $Z_{\text {in }}=1$ which is reached at a matching thickness $d=d_{m}$, matching frequency $f=f_{m}$, and minimum return loss $\mathrm{RL}=\mathrm{RL}_{\text {min }}$.

\section{Results and discussion}

SEM photographs of MnZn ferrite and carbon black powders can be found in Fig. 1. One may notice that the shape of particles is irregular (polyhedral and/or prismatic). The particle size of MnZn ferrite varies in the range of $0-80 \mu \mathrm{m}$, while that of carbon black varies in the interval $20-50 \mathrm{~nm}$.
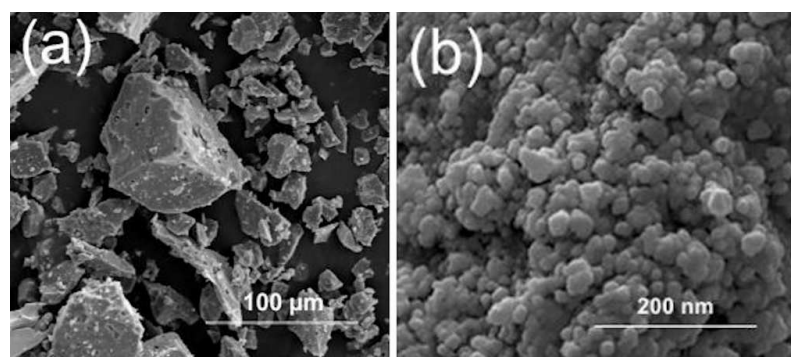

Fig. 1. SEM images of (a) MnZn ferrite and (b) carbon black.

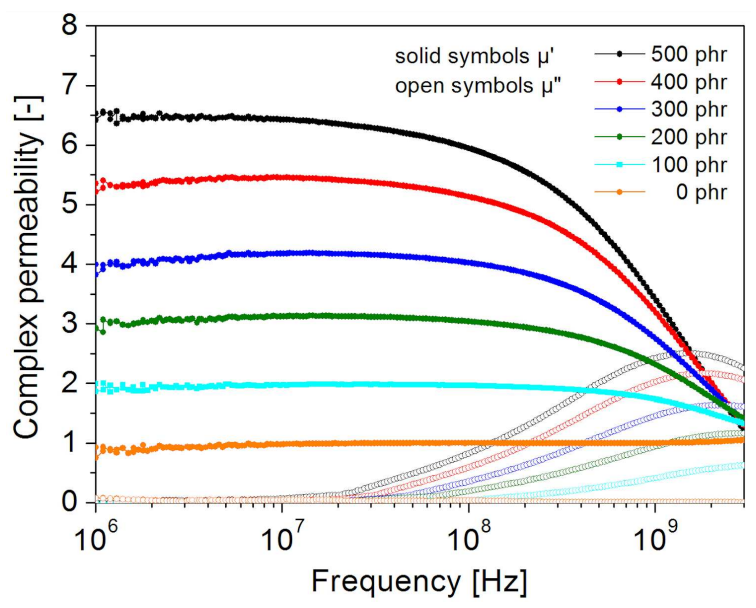

Fig. 2. Frequency dependences of real and imaginary parts of complex (relative) permeability for prepared $\mathrm{MnZn/CB/PVC} \mathrm{composite} \mathrm{materials.}$

The frequency responses of real $\mu^{\prime}$ and imaginary $\mu^{\prime \prime}$ parts of complex (relative) permeability $\mu=\mu^{\prime}-\mathrm{i} \mu^{\prime \prime}$ as a function of frequency $f(\mathrm{~Hz})$ for fabricated $\mathrm{MnZn/CB/NBR}$ composites can be found in Fig. 2. The parameter of curves is MnZn ferrite filler content (in "phr" units). The low-frequency value of real part $\mu^{\prime}$ of permeability at $f=1 \mathrm{MHz}$ decreases starting from 6.53 (for 500 phr composite sample) to about 0.99 (for $0 \mathrm{phr}$ composite sample). The vertex of imaginary part $\mu^{\prime \prime}$ goes down, and simultaneously the resonance frequency $f_{\text {res }}$, at which this vertex occurs, shifts towards a higher frequency region with the change of MnZn ferrite filler content in composite samples from 500 to $0 \mathrm{phr}$. Moreover, the frequency response of real part $\mu^{\prime}$ stagnates up to around $200 \mathrm{MHz}$, and then reduces to a low value. The highest drop of $\mu^{\prime}$ with frequency behind $200 \mathrm{MHz}$ has been observed in case of 500 phr composite sample. The $0 \mathrm{phr}$ composite sample did not show any frequency response of any part of $\mu$ because of its non-magnetic nature. The main mechanisms determining the $\mu$ of composites with magnetic filler comprise hysteresis loss, domain wall movement, spin precession, and eddy current effect [3]. In our case, the hysteresis loss due to the irreversible magnetization can be neglected as the composites were tested in Rayleigh region (of low magnetic fields), and at high frequencies. The domain wall movement and the spin precession are usually coupled with resonance phenomena in the permeability spectrum, namely the domain wall and the spin precession (or natural ferromagnetic) resonance. As shown in Fig. 2, the observed peaks in $\mu^{\prime \prime}-f$ dependencies correspond to the spin precession resonance only as the domain walls are unable to keep pace with ac electromagnetic field over about $10^{8} \mathrm{~Hz}$. Eddy current effect could also contribute to permeability loss due to high DC electrical conductivity $\sigma_{\mathrm{DC}}$ and low particle size of fillers $(\mathrm{MnZn}$ ferrite had $\sigma_{\mathrm{DC}} \approx 0.3 \mathrm{~S} / \mathrm{m}$, while $\mathrm{CB}$ had $\left.\sigma_{\mathrm{DC}} \approx 107 \mathrm{~S} / \mathrm{m}\right)$. The eddy currents cause the decrease in effective volume conducing to the $\mu$ thanks to the drop of the skin depth 


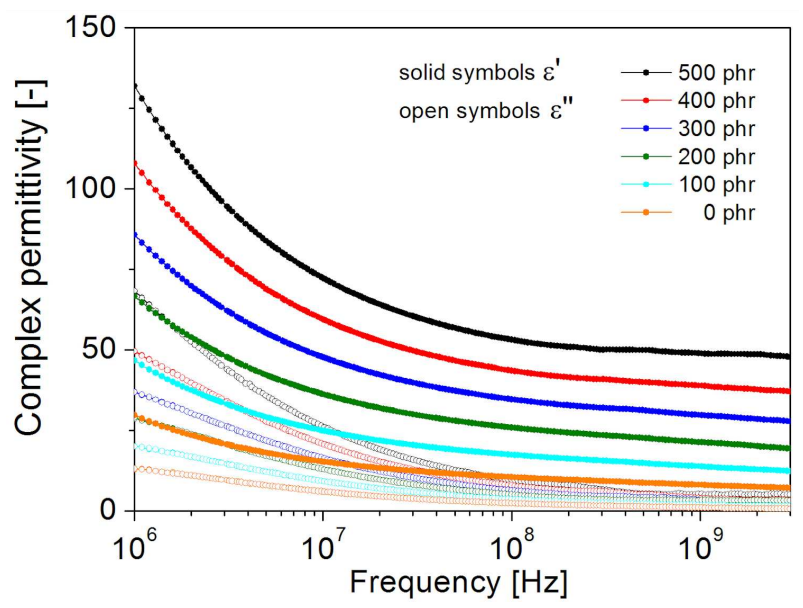

Fig. 3. Frequency dependences of real and imaginary parts of complex (relative) permittivity for prepared $\mathrm{MnZn/CB/PVC} \mathrm{composite} \mathrm{materials.}$

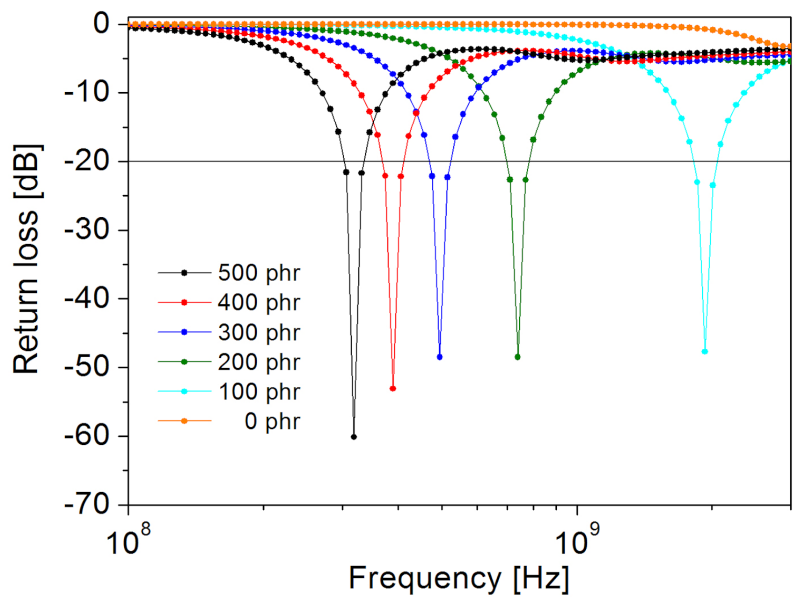

Fig. 4. Frequency dependences of return loss for prepared $\mathrm{MnZn} / \mathrm{CB} / \mathrm{PVC}$ composite materials.

$\left(\delta \approx \sqrt{1 / \pi f \sigma_{\mathrm{DC}} \mu_{0} \mu^{\prime}}\right.$ with $\mu_{0}$ as the permeability of free space). Then the reduction of $\mu$ happens behind the frequency, at which $\delta$ starts to be smaller than the sample thickness $(\approx 2 \mathrm{~mm})$.

Figure 3 depicts the frequency dependencies of real $\varepsilon^{\prime}$ and imaginary $\varepsilon^{\prime \prime}$ parts of complex (relative) permittivity $\varepsilon=\varepsilon^{\prime}-\mathrm{i} \varepsilon^{\prime \prime}$ for prepared composite materials. The steeply decrease of $\varepsilon^{\prime}$ is observed at frequencies up to about $10 \mathrm{MHz}$, and then $\varepsilon^{\prime}$ settles on a constant value. The same trend can be also observed in $\varepsilon^{\prime \prime}-f$ dependencies. This initial decrease of $\varepsilon^{\prime}$ with frequency may be due to semiconducting character of MnZn ferrite and conducting character of $\mathrm{CB}$ used as fillers in prepared composites. Note that when the content of MnZn ferrite filler in composites changes, $\varepsilon^{\prime}$ also drops at $f=1 \mathrm{MHz}$ : from $\varepsilon^{\prime} \approx 132$ for $500 \mathrm{phr}$ composite to $\varepsilon^{\prime} \approx 29$ for $0 \mathrm{phr}$ composite. The observed changes in the frequency responses of complex permittivity may be attributed to
TABLE I

Absorption parameters for prepared $\mathrm{MnZn} / \mathrm{CB} / \mathrm{PVC}$ composites.

\begin{tabular}{c|c|c|c|c}
\hline \hline $\begin{array}{c}\text { MnZn ferrite } \\
\text { content } \\
\text { in composite [phr] }\end{array}$ & $\begin{array}{c}d_{m} \\
{[\mathrm{~mm}]}\end{array}$ & $\begin{array}{c}f_{m} \\
{[\mathrm{MHz}]}\end{array}$ & $\begin{array}{c}\Delta f[\mathrm{MHz}] \\
\text { for }\end{array}$ & $\begin{array}{c}\mathrm{RL} \leq-20 \mathrm{~dB} \\
{[\mathrm{~dB}]}\end{array}$ \\
\hline 500 & 14.27 & 319 & 21.3 & -60.12 \\
400 & 14.08 & 390 & 42.2 & -53.10 \\
300 & 14.33 & 495 & 53.6 & -48.50 \\
200 & 13.38 & 739 & 73.1 & -48.52 \\
100 & 8.475 & 1931 & 157.5 & -47.71 \\
0 & - & - & - & -
\end{tabular}

various types of polarization mechanisms arising in filler (mainly interfacial polarization brought about by space charge accumulated at boundaries of filler particles dispersed in matrix), as well as polymeric matrix because of their dielectric character [4].

Figure 4 presents the frequency dependences of return loss RL $[\mathrm{dB}]$ for prepared composite materials. Table I summarizes the selected EM-wave absorption parameters: matching thickness $d_{m}$ of the absorber, matching frequency $f_{m}$, bandwidth $\Delta f$ for $\mathrm{RL} \leq-20 \mathrm{~dB}$, and a minimum value of return loss $\mathrm{RL}_{\text {min }}$ at $f=f_{m}$. With decrease of the content of MnZn ferrite filler in composites from 500 to $100 \mathrm{phr}$, one can see that the values of $f_{m}$, as well as $\Delta f$ increase in contrast to the values of $d_{m}$ and $\mathrm{RL}_{\text {min }}$. The obtained results are direct consequence of composite nature: the values of $f_{m}$ and $\Delta f$ increase due to the magnetic dilution of composite structure (with the decrease of MnZn ferrite filler content). This, in turns, leaded to the alternation of magnetic $(\mu)$ and dielectric $(\varepsilon)$ parameters, and therefore also absorption properties $\left(\mathrm{RL}, d_{m}, f_{m}, \Delta f, \mathrm{RL}_{\min }\right)$ of designed absorbers. The computed values of absorption parameters indicate that the prepared composite materials can be thought as suitable candidates for electromagnetic field shielding applications at frequencies above $300 \mathrm{MHz}$.

\section{Conclusions}

We have researched the frequency responses of material parameters such as complex permeability and permittivity of polymer-based composited with NBR as matrix, and $\mathrm{MnZn}$ ferrite and carbon black as fillers in the frequency range from $1 \mathrm{MHz}$ to $3 \mathrm{GHz}$. The permeability showed strong while permittivity weak dispersion character. On the basis of material parameters responses we have calculated the return loss and also selected electromagnetic absorption parameters. The absorption peak (the minimum of return loss) shifted towards higher frequencies with the drop of MnZn ferrite in composites. The synthesized composites may be utilized as elastomeric electromagnetic field shields. 


\section{Acknowledgments}

This work was supported by the Slovak Research and Development Agency under the contracts no. APVV-160059 and APVV-15-0257, by the Scientific Grant Agency of the Ministry of Education, Science, Research and Sport of the Slovak Republic (VEGA), under project no. $1 / 0405 / 16$, and by the University Science Park SUT Bratislava (project IMTS: 26240220084). The authors would also like to thank for financial assistance from the STU Grant scheme for Support of Excellent Teams of Young Researchers.

\section{References}

[1] Y. Pang, Y. Li, J. Wang, M. Yan, S. Qu, S. Xia, Z. Xu, Carbon 147, 112 (2019).

[2] S. Sankaran, K. Deshmukh, M.B. Ahamed, S.K.K. Pasha, Compos. Part A 114, 49 (2018).

[3] R. Dosoudil, M. Ušáková, A. Grusková, J. Sláma, IEEE Trans. Magn. 50, 2800204 (2014).

[4] S.Y. Tong, J.M. Wu, M.J. Tung, W.S. Ko, Y.T. Huang, Y.P. Wang, J. Alloys Compd. 525, 143 (2012). 